Brazilian Journal

of Chemical

ISSN 0104-6632

Engineering

\title{
REAL TIME AND IN SITU NEAR-INFRARED SPECTROSCOPY (NIRS) FOR QUANTITATIVE MONITORING OF BIOMASS, GLUCOSE, ETHANOL AND GLYCERINE CONCENTRATIONS IN AN ALCOHOLIC FERMENTATION
}

\author{
R. J. A. do Nascimento*, G. R. de Macedo, E. S. dos Santos and J. A. de Oliveira \\ Biochemical Engineering Laboratory, Department of Chemical Engineering, Federal University of Rio Grande do Norte, \\ Natal, Rio Grande do Norte, Brazil. \\ Phone: +558432153769 (Ext. 214), Fax: +55 8432153770 \\ E-mail: ruthjessica@gmail.com, gomacedo@eq.ufrn.br, everaldo@eq.ufrn.br, jackson@eq.ufrn.br
}

(Submitted: June 2, 2015; Revised: January 22, 2016; Accepted: March 15, 2016)

\begin{abstract}
This study investigates the application of NIR spectroscopy for real-time monitoring of process parameters in alcoholic fermentations. For this purpose, fermentation batches using Saccharomyces cerevisiae were carried out and monitored in situ by an immersed NIRS probe. Samples were collected throughout the fermentations, and concentrations of biomass, glucose, ethanol and glycerin were analyzed by reference methods and multivariate data analyses such as PCA and PLSR. PCA was used to investigate data variability and to select samples for calibration and for external validation sets. A PLSR model for biomass yielded $\mathrm{R}^{2}$ and RMSEP values of 0.99 and 0.276 g.L. $\mathrm{L}^{-1}$, respectively. For glucose, the carbon source, the PLSR model yielded an $\mathrm{R}^{2}$ of 0.95 and an RMSEP of 7.14 g.L. $\mathrm{L}^{-1}$. The PLSR model for ethanol was characterized by an $\mathrm{R}^{2}$ of 0.95 and an RMSEP of 0.06 g.L $\mathrm{L}^{-1}$. For glycerin, a highly accurate PLSR model with an $\mathrm{R}^{2}$ of 0.98 and an RMSEP of 0.116 g.L-1 was obtained. These results indicate that an in situ NIRS probe is suitable for real-time monitoring of important parameters in alcoholic fermentations.

Keywords: near-infrared spectroscopy; monitoring; alcoholic fermentation.
\end{abstract}

\section{INTRODUCTION}

Efficient real time monitoring of bioprocesses allows for improved process modeling and control (Schügerl, 2001), which can lead to increased process yield, productivity and reproducibility (Alves-Raush et al., 2014). Therefore, instrumentation capable of real-time monitoring is essential for bioprocess optimization.

The yeast Saccharomyces cerevisiae is the most frequently used microorganism for ethanol production in the energy and beverage industries. During fermentation, culture broth samples are routinely taken to monitor and measure specific process parameters. Physical parameters such as temperature, $\mathrm{pH}$, dissolved oxygen, liquid level and foam level are commonly monitored by on-line sensors (Tamburini et al., 2003), but critical process parameters such as substrate consumption, cell concentration and final product concentration are typically measured with off-line and time-consuming methods. Off-line measurements do not allow process control to be implemented and reduce process efficiency (Blanco et al., 2005).

Analytical spectrometry-based methods have been used to monitor ethanol fermentations (Blanco et al., 2004; Finn et al., 2006; Burratti et al., 2011), as these methods are

*To whom correspondence should be addressed 
fast and non-destructive. In addition, these methods allow simultaneous analysis of multiple parameters (Ferreira et al., 1999) without requiring sample pretreatment. Nearinfrared spectroscopy (NIRS) is an important technique: fiber optic probes connected directly to the process enable simultaneous real-time and in situ monitoring of several parameters quickly and safely. Although the NIR sensors are able to withstand sterilization procedures, they are severely disturbed by the bubbles generated in vigorously aerated and mixed processes (Alves-Rausch et al., 2014).

The NIR absorbance region corresponds to overtones and combinations of the fundamental vibrations of $\mathrm{O}-\mathrm{H}, \mathrm{N}-\mathrm{H}$ and $\mathrm{C}-\mathrm{H}$ bounds (Buratti et al., 2011). These characteristics result in spectra with broad and overlapping bands (Alves-Raush et al., 2014). First and second derivatives are used to "pretreat" NIR spectra to enhance spectral features and to reduce baseline offsets resulting from light scattering due to increases in cell concentration (Tamburini et al., 2003).

NIR spectroscopy has been used to monitor microbial fermentations, and many studies have used NIR sensors to monitor off-line and at-line. For at-line monitoring, the sample is transported through an external line to the probe, where the measurement is performed at a sampling rate often inadequate for process control objectives (Blanco et al., 2004; Blanco et al., 2006; Finn et al., 2006; GonzálezSáiz et al., 2008; Fernandéz-Novales et al., 2008; Xie et al., 2009; Burratti et al., 2001). Grassi et al. (2014) used NIRS in off-line mode to monitor $\mathrm{pH}$, biomass and soluble solid content (Brix) in a wort fermentation. By using off-line NIRS equipment, Grassi et al. required the use of centrifugation prior to analyses. Liang et al. (2013) reported the use of at-line NIRS to monitor glutamate, glucose, lactate and alanine concentrations in a glutamate fermentation. They concluded that it is feasible to use NIRS to determine compound concentrations in the fermentation industry. Studies using NIR spectroscopy to monitor analyte concentrations in fermentation with other microorganisms can be found in the literature (Crowley et al., 2005; Roychoudhury et al., 2006; González-Sáiz et al., 2008; Jiang et al., 2012; Liang et al., 2013). To date, the use of on-line NIR spectrophotometers to monitor alcoholic fermentations has been limited and has been described in relatively few studies, especially compared to off-line and at-line monitoring.

Other spectroscopic techniques have also been used to monitor bioprocesses. Fayolle et al. (2000) used midinfrared spectroscopy (mid-IR) to monitor sugars, ethanol and organic acids concentrations on-line in alcoholic and lactic acid fermentations. They measured the contents of glucose, fructose, galactose, lactose, ethanol and lactic acid but reported that predictions of concentrations were not accurate enough to guarantee good on-line monitoring. Cozzolino et al. (2006) found that NIRS and visible spectroscopy, along with chemometrics, can be used to monitor the progress of red wine fermentations. The authors concluded that visible and NIR spectroscopies provide good solutions to accurately monitor the progress of red wine fermentations without requiring any chemical analyses. NIRS and MIRS were used by Egidio et al. (2010) to monitor the main chemical parameters in red wine fermentations. The monitoring of the bioprocess was offline, and the authors concluded that NIRS and MIRS were able to measure the concentrations of red wine analytes such as glucose, fructose, ethanol, glycerol, total phenolics, total anthocyanins and total flavonoids. Hakemeyer et al. (2013) used NIRS and 2-D fluorescence spectroscopy to monitor the chemical composition of culture media during storage, concluding that both spectroscopic methods were able to predict chemical composition changes over time with good accuracy.

Multivariate techniques allow the use of spectral information both qualitatively and quantitatively (AlvesRaush et al., 2014). Quantitative multivariate analysis uses multilinear regression methods to relate a matrix $\mathbf{X}$ of NIRS data with a matrix $\mathbf{Y}$ of reference concentration data (Naes et al., 2002). Partial least squares regression (PLSR) is a multilinear regression method that may be used to obtain quantitative calibration models from matrices $\mathbf{X}$ and $\mathbf{Y}$. The models created by PLSR can be used to predict analyte concentrations in systems at lab or industrial scales.

The aim of the present study is to evaluate in situ NIRS monitoring of an alcoholic fermentation process using $S$. cerevisae. This study is also intended to develop predictive PLSR models for the quantitative prediction of glucose, biomass, ethanol and glycerine concentrations. The regression models developed here may be used for realtime bioprocess monitoring and control.

\section{MATERIALS AND METHODS}

\section{Yeast and cultivation conditions}

The Saccharomyces cerevisiae CAT-1 fermentec strain was obtained from an Estivas ${ }^{\circledR}$ industrial plant located in the Arês municipality in the state of Rio Grande do Norte, Brazil. The strain was maintained on Petri dishes containing YEPD medium at $4{ }^{\circ} \mathrm{C}$ with the following nutrient concentrations: $10 \mathrm{~g} / \mathrm{L}$ of yeast extract, $20 \mathrm{~g} / \mathrm{L}$ of peptone, $20 \mathrm{~g} / \mathrm{L}$ of glucose and $15 \mathrm{~g} / \mathrm{L}$ of agar.

Pre-inoculum: Pre-cultures of yeast cells were grown in two 250-mL Erlenmeyer flasks containing $150 \mathrm{~mL}$ of cultivation medium (in $\mathrm{g} \mathrm{L}^{-1}$ : glucose 30 ; yeast extract 5; $\left(\mathrm{NH}_{4}\right)_{2} \mathrm{SO}_{4} 10 ; \mathrm{KH}_{2} \mathrm{PO}_{4} 4.5 ; \mathrm{MgSO}_{4} .7 \mathrm{H}_{2} \mathrm{O} 1 ; \mathrm{ZnSO}_{4} .7 \mathrm{H}_{2} \mathrm{O}$ 0.65 and $\mathrm{pH}$ adjusted to 5.0). Each Erlenmeyer flask was inoculated with Saccharomyces cerevisiae and incubated in a rotary incubator at $30^{\circ} \mathrm{C}, 150 \mathrm{rpm}$ for $20 \mathrm{~h}$. After $20 \mathrm{~h}$ of cultivation, the contents of the Erlenmeyer flasks were transferred to three $50 \mathrm{~mL}$ centrifuge tubes and centrifuged at $3000 \mathrm{rpm}$ for $10 \mathrm{~min}$. The supernatant was discarded, and 
the cells in each of the three flasks were resuspended in 50 $\mathrm{mL}$ of fresh, sterile medium. The resuspended cells (total volume of $150 \mathrm{~mL}$ ) were transferred to a sterile inoculator flask, forming the initial biomass used for fermentation tests.

Bioreactor: Cultivations for ethanol production were performed in a BIOSTAT $\mathrm{B}^{\circledR}$ (B. Braun Biotech International) reactor with a volumetric capacity of $2 \mathrm{~L}$ and a working volume of $1.65 \mathrm{~L}$. The composition of the cultivation medium used was the same as the medium used for the pre-inoculum. The temperature was maintained at $30{ }^{\circ} \mathrm{C}$. The $\mathrm{pH}$ setpoint was set at 5.0 , and cultivation conditions were established to maintain an environment of restricted aerobiosis without aeration. Agitation was performed at $150 \mathrm{rpm}$.

\section{Sampling}

The samples used to build the calibration model were obtained from five different fermentations. Samples were taken with a $10 \mathrm{~mL}$ plastic syringe connected to the bioreactor collection tube. Batch 1 was sampled every hour; the other batches were sampled every half hour. Each sample was divided into two aliquots. One aliquot was used to determine cell concentrations, and the second aliquot was divided into $2 \mathrm{~mL}$ Eppendorf ${ }^{\circledR}$ microcentrifuge tubes. These microcentrifuge tubes were centrifuged for $15 \mathrm{~min}$ at 14,000 $\mathrm{rcf}$ in an Eppendorf centrifuge (model $5415 \mathrm{D}$ ). The supernatant was filtered through a $0.22 \mu \mathrm{m}$ PES membrane and frozen for later reference analysis of glucose, glycerine and ethanol concentrations by HPLC.

\section{Reference Methods}

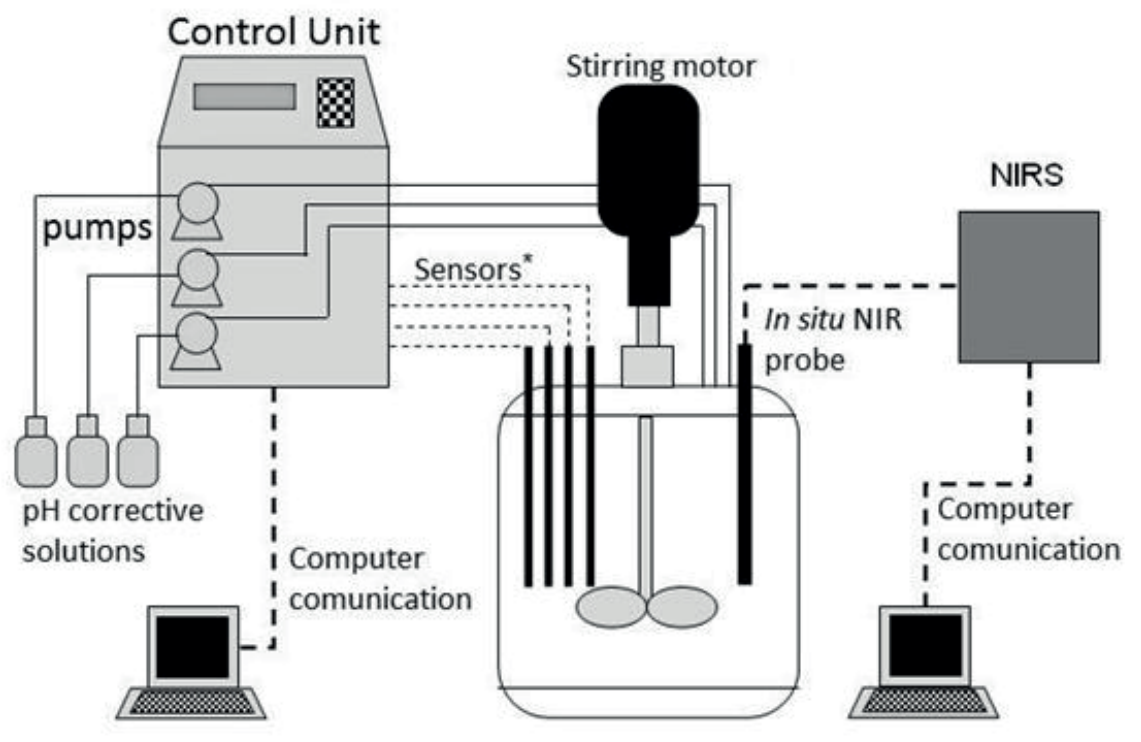

HPLC - the levels of glucose, ethanol and glycerine were determined by HPLC (Shimadzu LC-10A series). Analyses were performed at $65{ }^{\circ} \mathrm{C}$ on a Shimadzu SCR$101 \mathrm{H}$ column with a refractive index detector (RID-10A). A 5 mmol. $\mathrm{L}^{-1}$ sulfuric acid solution was used as the mobile phase with a flow rate of $0.6 \mathrm{~mL} \cdot \mathrm{min}^{-1}$.

UV Absorption - The absorbances of aliquots intended for cell concentration measurements were determined at $600 \mathrm{~nm}$ in a 10 UV Genesys spectrophotometer. Sample cell concentrations were determined from the absorbance data using a previously obtained linear regression equation.

\section{NIR spectral measurements}

NIR spectra of cultivation samples were acquired using a Thermo-Nicolet Antaris II FT-NIR spectrophotometer equipped with a probe connected with optical fiber cables. The transflectance adapter is attached to the sensor, enabling the optical path to be adjusted from $1.5 \mathrm{~mm}$ to 10 $\mathrm{mm}$. In this study, the smallest optical path $(1.5 \mathrm{~mm})$ was used. The blank spectrum, which was used as a reference during calculation of sample absorbance values, was measured automatically using an internal white standard as the background spectrum. Spectral scans were performed over the entire NIR range (10000 to $4000 \mathrm{~cm}^{-1}$ ) with an optical resolution of $8 \mathrm{~cm}^{-1}$. Each recorded spectrum was the average of 64 scans, and each spectrum was stored and processed with TQ ANALYST 8 (Thermo Scientific). Chemometric analysis was performed using the same software package. In cultivations for ethanol production, the sensor was directly attached to the bioreactor, and spectra were acquired in real time. Figure 1 shows a schematic diagram of the bioreactor used in this study. 


\section{Data analysis}

In highly aerated processes, air bubbles passing in front of the NIR probe take the place of the liquid sample, reducing the measured absorbance (Alves-Raush et al., 2014). Because alcoholic fermentations do not require aeration, the use of spectral filters to reduce the effect of air bubbles was not necessary.

Five batches were monitored in situ, and samples were taken for off-line reference analysis. Table 1 shows the number of samples used to generate each calibration model as well as the respective concentration range of each model.

Principal Component Analysis (PCA) was used to investigate the total variability of samples and to select data for calibration and external validation sets. PCA is a multivariate analysis technique that aims to minimize the original data dimensionality into a small number of variables called principal components. In the PCA score plot, observations that are closer to one another have similar characteristics (Cordella, 2012). Therefore, PCA is a useful tool for extracting information from data sets.

Calibration models were generated for glucose, ethanol, glycerin and cell density in TQ ANALYST 8 by applying PLSR to the spectral data and analyte reference concentrations. The PLS algorithm has been described in the literature (Geladi and Kowalsky, 1986; Svante, 1995; Naes et al., 2002) and determines the relationship between the X matrix (spectral data) and the Y matrix (reference analyte concentration). For model validation, a batch was chosen by PCA and kept out of the calibration process to be used as an external validation set. Table 1 shows the number of samples and the sample ranges used in the calibration models.

Table 1. Concentration range (g. $\left.\mathrm{L}^{-1}\right)$ and number of samples included in the calibration and external validation sets.

\begin{tabular}{ccccc}
\hline & \multicolumn{4}{c}{ Experimental data - number of points } \\
\cline { 2 - 5 } & Biomass & Glucose & Ethanol & Glycerine \\
\hline Calibration & 58 & 53 & 45 & 48 \\
\hline Validation & 17 & 17 & 16 & 12 \\
\hline $\begin{array}{c}\text { Concentration range } \\
\left(\mathrm{g} . \mathrm{L}^{-1}\right)\end{array}$ & $0.56-5.85$ & $0.30-39.61$ & $0.30-14.80$ & $0.00-1.16$ \\
\hline
\end{tabular}

Calibration models were constructed using the "leaveone-out" cross-validation procedure. The optimal number of latent (factors) variables was determined by minimizing the sum of residual squared errors of prediction (PRESS), Eq. 1 (Randall, 1999; Blanco et al., 2004; Coffey and Cooley, 1999) and minimizing the root mean squared errors of prediction (Eq. 2) obtained by external validation.

$$
\operatorname{PRESS}=\sum_{\mathrm{i}=1}^{\mathrm{m}}\left(\mathrm{y}_{\mathrm{i}}^{\mathrm{NIR}}-\mathrm{y}_{\mathrm{i}}^{\mathrm{REF}}\right)^{2}
$$

Where $m$ is the number of samples, $y^{\mathrm{REF}}$ is the value of the reference concentration, and $\mathrm{y}^{\mathrm{NIR}}$ is the concentration calculated by the NIRS calibration model.

The accuracies of the calibration models are expressed as the average root mean square error detailed in Eq. 2. These accuracies are referred to as RMSEC for calibration, RMSECV for cross-validation (internal validation) and RMSEP for external validation.

$$
R M S E=\sqrt{\frac{\left(\sum_{i=1}^{m}\left(\mathrm{y}_{i}^{N I R}-\mathrm{y}_{i}^{R E F}\right)\right)^{2}}{m}}
$$

\section{RESULTS AND DISCUSSION}

Quantitative calibration models were generated for glucose, ethanol, glycerine and cell concentrations. Online monitoring of these parameters provides valuable information, allowing the evolution of fermentations in industrial environments to be followed. The calibration models provide predicted values of parameters and may be used to implement control loops capable of reducing substrate feeds and increasing end-product quality and process yield.

To obtain robust models and to implement a real-time monitoring system for alcoholic fermentations, calibration and validation procedures were performed to identify the model that allows for the best quantitative analysis of biomass, substrate and product concentration. The chemometric approach was applied by using near-infrared spectroscopy and reference analytical techniques. The calibration procedure must guarantee that all variability in the process is captured, so the calibration set should include several batches to introduce inter-batch variability into the model. The external validation procedure consists of using a set of validation samples that do not belong to the calibration set. This method facilitates the evaluation of model robustness for predicting analyte concentrations in future fermentation batches. New spectra will be in the design space covered by the calibration procedure. 


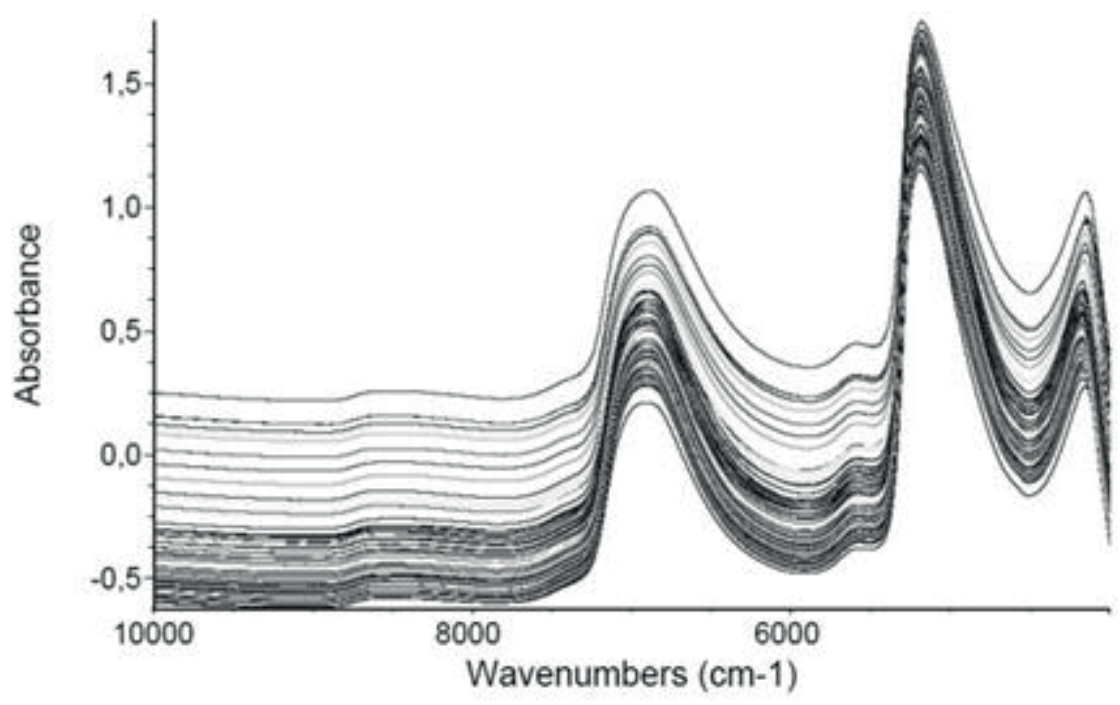

Figure 2. NIRS acquired spectra for samples of all fermentations.

Sample spectra acquired with the NIR sensor are shown in Figure 2. It is noted that the spectra do not provide information in a clear manner, as NIR spectra are characterized by overlapping absorption bands and light scattering differences caused by increases in cell concentration (Hall et al., 1996, Nishii et al., 2012). To enhance spectral features and to compensate for baseline shifts and light scattering differences, the original spectra need to be subjected to pretreatments before being used to construct calibration models (Finn et al., 2006). The first and the second derivatives are used to solve the problem of overlapping peaks and to eliminate the baseline shift associated with increasing biomass concentration (Petersen et al., 2009). The Savitzky-Golay smoothing filter allows the random noise to be reduced. Figure 3 illustrates the spectra after pretreatment with first and second derivatives.
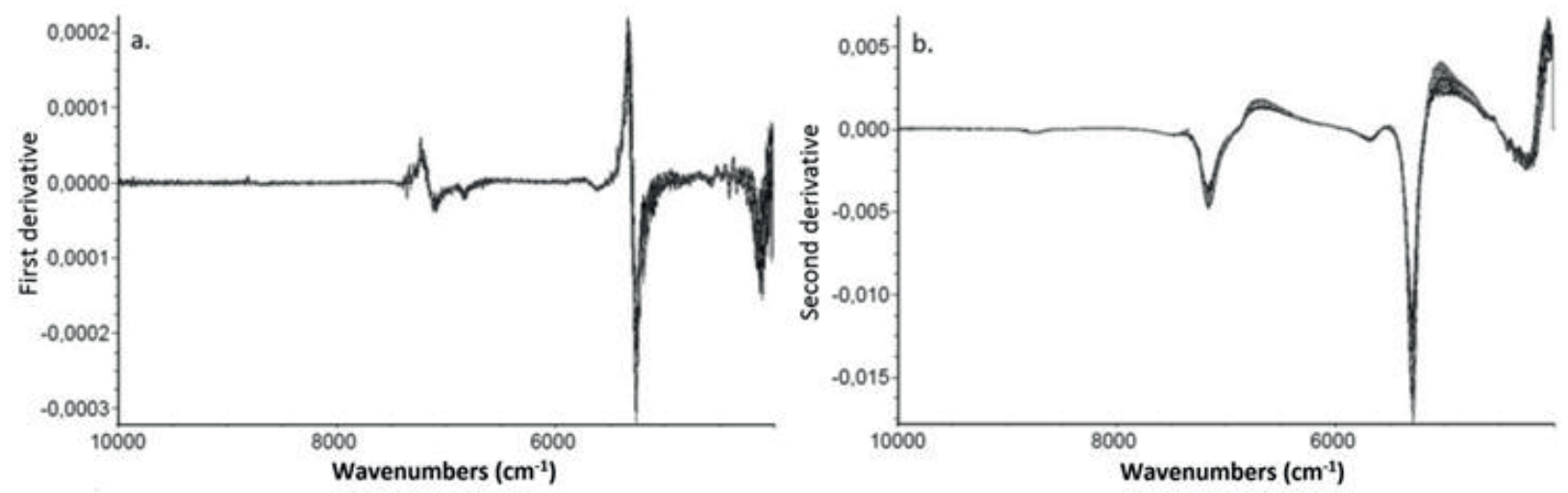

Figure 3. (a) NIR spectra of samples using first derivative and Savitzky-Golay smoothing filter; (b) NIR spectra of samples using second derivative and Savitzky-Golay smoothing filter.

To obtain an adequate model, it is important that calibration data include all of the variations observed throughout the process. In this study, the data from all fermentation samples were mean centered prior PCA, and a score plot was built (Figure 4) to determine which data set was more significant for model calibration and which data set could be used for external validation. With PCA, it was possible to verify that the samples presented distinct characteristics throughout the bioprocess, including differences in changes in biomass, substrate, and product concentrations. In the score plot (Figure 4); experimental data for batches 1 and 3 were clustered together, indicating the similarity of these samples. The same behavior was observed for batches 2 and 5. Batch 4 did not cluster with 
any of the other batches, indicating that this batch contains samples with features distinct from the other batches. Therefore, batches 1 to 4 were used for model calibration. This data set contains the greatest number of samples with different characteristics, increasing the variability of the model prediction. Batch 5 was chosen for external validation, as this experimental run was a completely independent data set whose features were contained within the calibration data set.

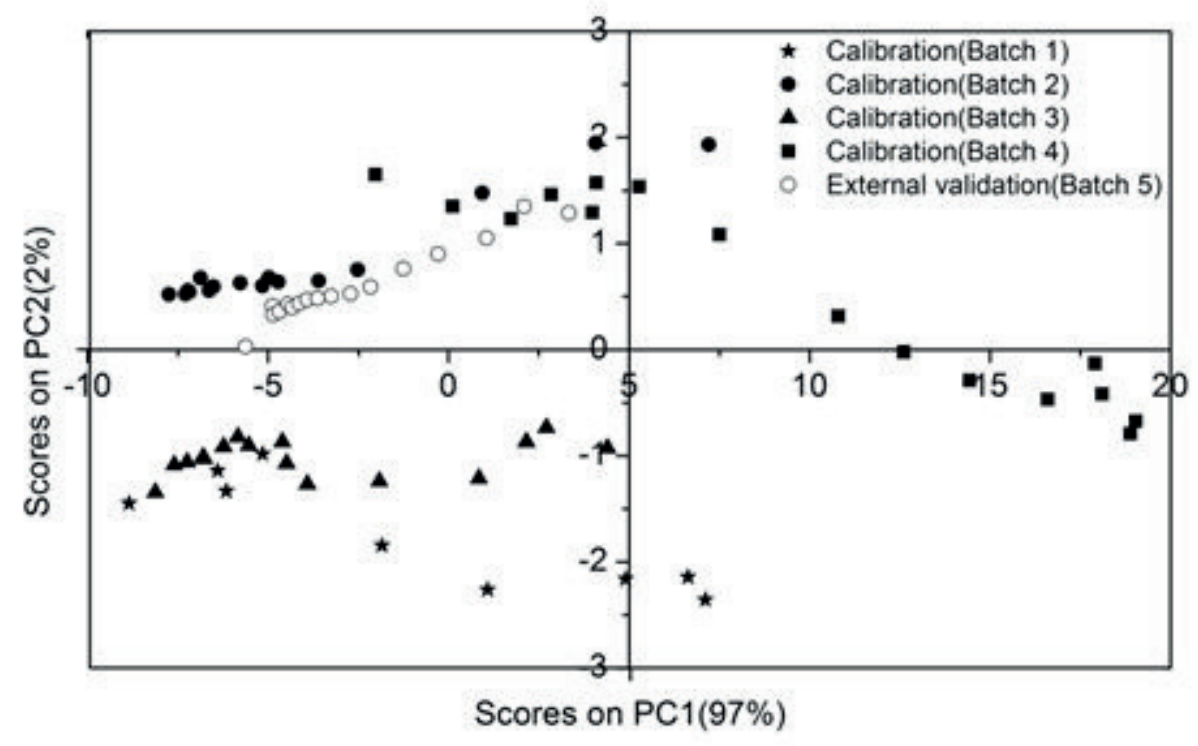

Figure 4. PCA score plot of the calibration and external validation samples. The solid symbols denote calibration samples and the hollow symbols represent external validation samples.

TQ ANALYST 8 was used to select the regions of the spectra where spectral information either varied or correlated with changes in concentrations of the analyzed compounds. The NIRS wavelength range influences the results of the calibration model. This is because only spectral information that changes or correlates with concentration is expected to be used in model construction (Hongqiang and Hongzhang, 2008). PLS calibration models using selected NIR regions were constructed, and the prediction results obtained from these models are summarized in Table 2.

Table 2. Calibration and validation results of PLS models for fermentation substrate and products using different pretreatments and the smoothing filter of Saviztky-Golay.

\begin{tabular}{|c|c|c|c|c|c|c|c|c|c|c|c|}
\hline \multirow[b]{2}{*}{ Analyte } & \multirow[b]{2}{*}{ Pretreatment } & \multirow[b]{2}{*}{ Filter } & \multirow{2}{*}{$\begin{array}{c}\text { Range } \\
\left(\mathrm{cm}^{-1}\right)\end{array}$} & \multirow[b]{2}{*}{ Factor } & \multicolumn{2}{|c|}{ Calibration } & \multicolumn{2}{|c|}{ Cross Validation } & \multicolumn{3}{|c|}{ External Validation } \\
\hline & & & & & $\mathbf{R}^{2}$ & $\begin{array}{c}\text { RMSEC } \\
\left(\mathrm{g} \cdot \mathrm{L}^{-1}\right)\end{array}$ & $\mathbf{R}^{2}$ & $\begin{array}{c}\text { RMSECV } \\
\left(\text { g.L.L }{ }^{-1}\right)\end{array}$ & $\mathbf{R}^{2}$ & $\begin{array}{c}\text { RMSEP } \\
\left(\text { g.L } L^{-1}\right)\end{array}$ & Bias \\
\hline Biomass & $1^{\text {a }}$ derivative & $\mathrm{SG}^{\mathrm{a}}$ & $\begin{array}{c}5916.54- \\
4389.19\end{array}$ & 7 & 0.992 & 0.230 & 0.978 & 0.376 & 0.983 & 0.276 & -0.10049 \\
\hline Glucose & None & $\mathrm{SG}^{\mathrm{a}}$ & $\begin{array}{c}7328.17- \\
4130.78\end{array}$ & 9 & 0.955 & 3.52 & 0.920 & 4.65 & 0.940 & 7.14 & 7.04987 \\
\hline Ethanol & $1^{\mathrm{a}}$ derivative & $\mathrm{SG}^{\mathrm{a}}$ & $\begin{array}{c}8261.55- \\
4327.48\end{array}$ & 6 & 0.952 & 1.21 & 0.911 & 1.64 & 0.915 & 2.36 & -2.70556 \\
\hline Glycerin & $2^{\mathrm{a}}$ derivative & $\mathrm{SG}^{\mathrm{a}}$ & $\begin{array}{c}4454.76- \\
4269.63\end{array}$ & 2 & 0.979 & 0.0759 & 0.970 & 0.0902 & 0.937 & 0.116 & 0.08553 \\
\hline
\end{tabular}

${ }^{a}$ Savitzky-Golay smoothing filter 
The fermentations were planned to be performed under similar conditions. However, each experimental run was carried out independently. Initial concentrations of biomass were inevitably different for each experimental run, and analyte concentrations evolved differently throughout each fermentation. These differences ensure that these data sets are different and independent. Furthermore, the samples used for external validation were within the calibration range, but belonged to an independent batch that was not included in the calibration set.

To check the predictive ability of the models, an external validation was performed: new samples that did not belong to the calibration set were used to evaluate the previously obtained models.

Figure 5 shows the calibration and validation results for biomass, glucose, ethanol and glycerine. The calibration and validation values remained close to the line of perfect
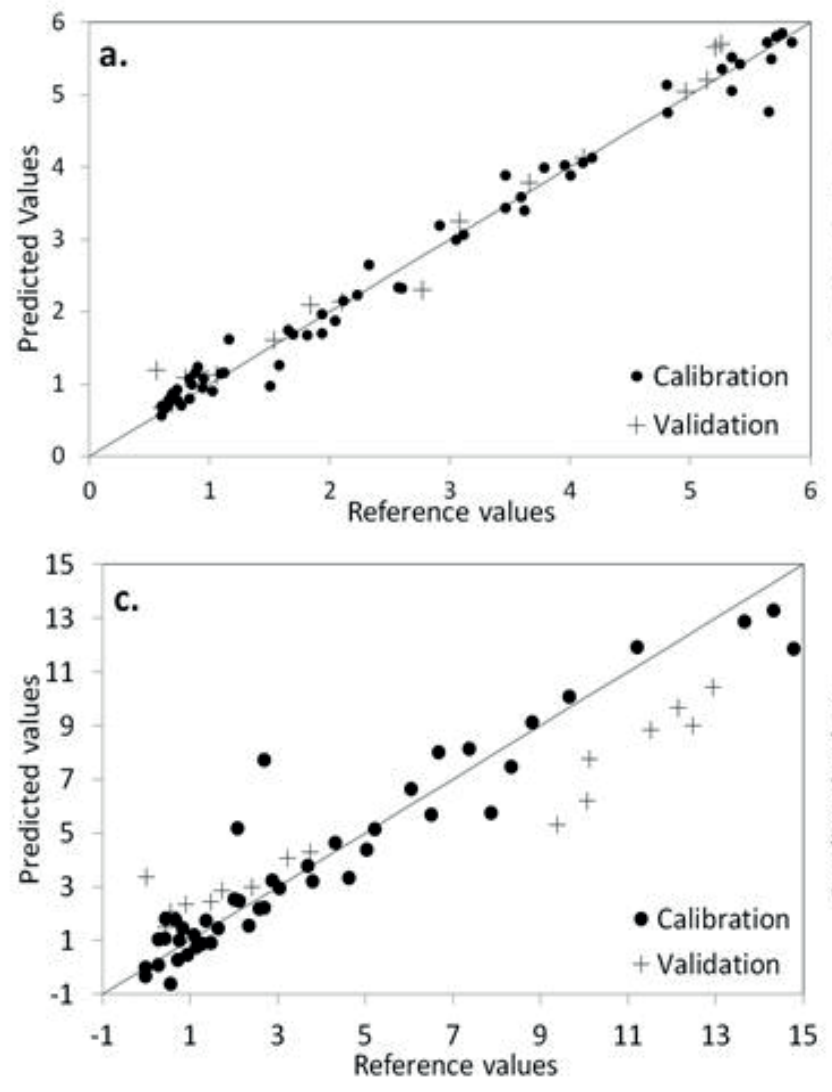

correlation, indicating that analyte concentrations in the broth were modeled successfully. The concentrations of biomass, glucose, ethanol and glycerine predicted more accurately when the Savitzky-Golay smoothing filter was used, as the filter was able to reduce the random noise present in the spectra (Hongqiang and Hongzhang, 2008) and make the data easier to interpret.

The best model for biomass was obtained using a first derivative pretreatment and the Saviztky-Golay smoothing filter, with RMSEC and RMSEP values of 0.230 g.. $\mathrm{L}^{-1}$ and 0.276 g.L. $\mathrm{L}^{-1}$, respectively. The RMSEP and RMSEC values are very close, indicating that the obtained calibration model retained its predictive capability for the new samples not belonging to the calibration set. Gonzáles-Sáiz et al. (2007) used NIR to monitor the alcoholic fermentation of onion juice, obtaining RMSEC and RMSEP values of 0.104 g.L $\mathrm{L}^{-1}$ and $0.157 \mathrm{~g} . \mathrm{L}^{-1}$, respectively.
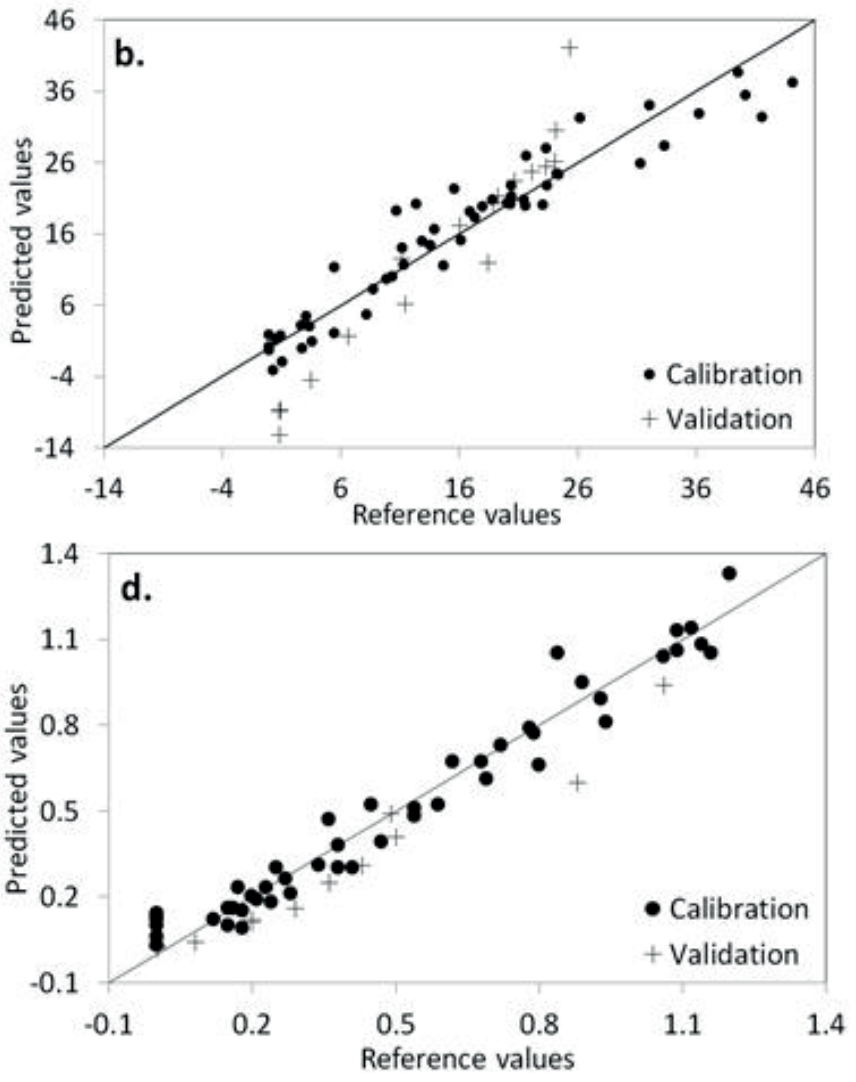

Figure 5. PLS regression of predicted vs. actual concentration values of: (a.) Biomass, (b.) glucose, (c.) ethanol and (d.) glycerine. The results for the best models are plotted.

The best results for glucose and ethanol prediction were obtained using the Savitzky-Golay smoothing filter. The best results for glucose prediction were obtained without pretreatment, while the best results for ethanol prediction were obtained with a first derivative pretreatment. For glucose, the correlation coefficient was approximately
0.96, and RMSEP and RMSEC values were 7.14 g.L.- and 4.65 g. $\mathrm{L}^{-1}$, respectively. The calibration model obtained for glucose had high RMSEC and RMSEP values, indicating a model with lower predictive power and reduced ability to quantify residual glucose at the ends of fermentations. The ethanol model had an RMSEC value of $1.21 \mathrm{~g} . \mathrm{L}^{-1}$ and an 
RMSEP value of 2.36 g.L $\mathrm{L}^{-1}$. Meng-Lin et al. (2009) used NIR to determine the ethanol concentration of an anaerobic $\mathrm{H}_{2}$-producing bioreactor effluent; RMSEC and RMSEP values for this model were $25.8 \mathrm{mg} / \mathrm{L}$ and $39.7 \mathrm{mg} / \mathrm{L}$, respectively. These values are lower than the values for the model in this work, but the models generated by Meng-Lin et al. (2009) were obtained with a large number of factors and may be overfitted. The fact that glucose and ethanol absorb in the same region (Nishii et al., 2012) lowered the prediction efficiency of these two analyte concentration models (Arnold et al., 2003; Finn et al., 2006). Due to this overlap in absorption, many factors are required in the glucose and ethanol prediction models. This result may mean that the best approach for describing the dataset is to construct nonlinear models, as nonlinear models require a larger number of factors to describe the nonlinearities present in the dataset (Geladi and Kowalski, 1986).

The best model for glycerine was generated using a second derivative pretreatment and the Savitzky-Golay smoothing filter, with RMSEC and RMSEP values of 0.0759 g.L. ${ }^{-1}$ and 0.116 g.L. ${ }^{-1}$, respectively. The determination of glycerol in fermentation broth has also been attempted by Hall et al. (1996), who reported a correlation coefficient and standard error of prediction (SEP) of 0.996 and 2.1, respectively. Although Hall et al. (1996) reported a higher coefficient of regression than this study, the authors also reported a higher standard error of prediction.

\section{Prediction}

The calibration models were developed using data from batches 1 to 4 and batch 5 was used for external validation. Figure 6 shows the evolution of the fermentation batch used to validate the proposed models.

The fermentation process was monitored for $8 \mathrm{~h}$. The graph shows the temporal evolution of glucose, ethanol, glycerine and biomass concentrations. Glucose concentration decreased throughout the process, as the microorganisms consume the sugars to produce ethanol, glycerine and additional biomass. Near infrared spectroscopy was able to monitor the fermentation process in real-time and in situ, but glucose and ethanol concentrations were poorly predicted at the end of the fermentation run, likely due to the fact that glucose and ethanol absorb in the same NIR region. This overlap in absorption lowers the model predictability. Despite this limitation, the glucose and ethanol models were able to follow analyte trajectories throughout the fermentation process. The models obtained for glycerin and biomass were adequate: for these two components; the predicted values were very close to those obtained via the reference method.
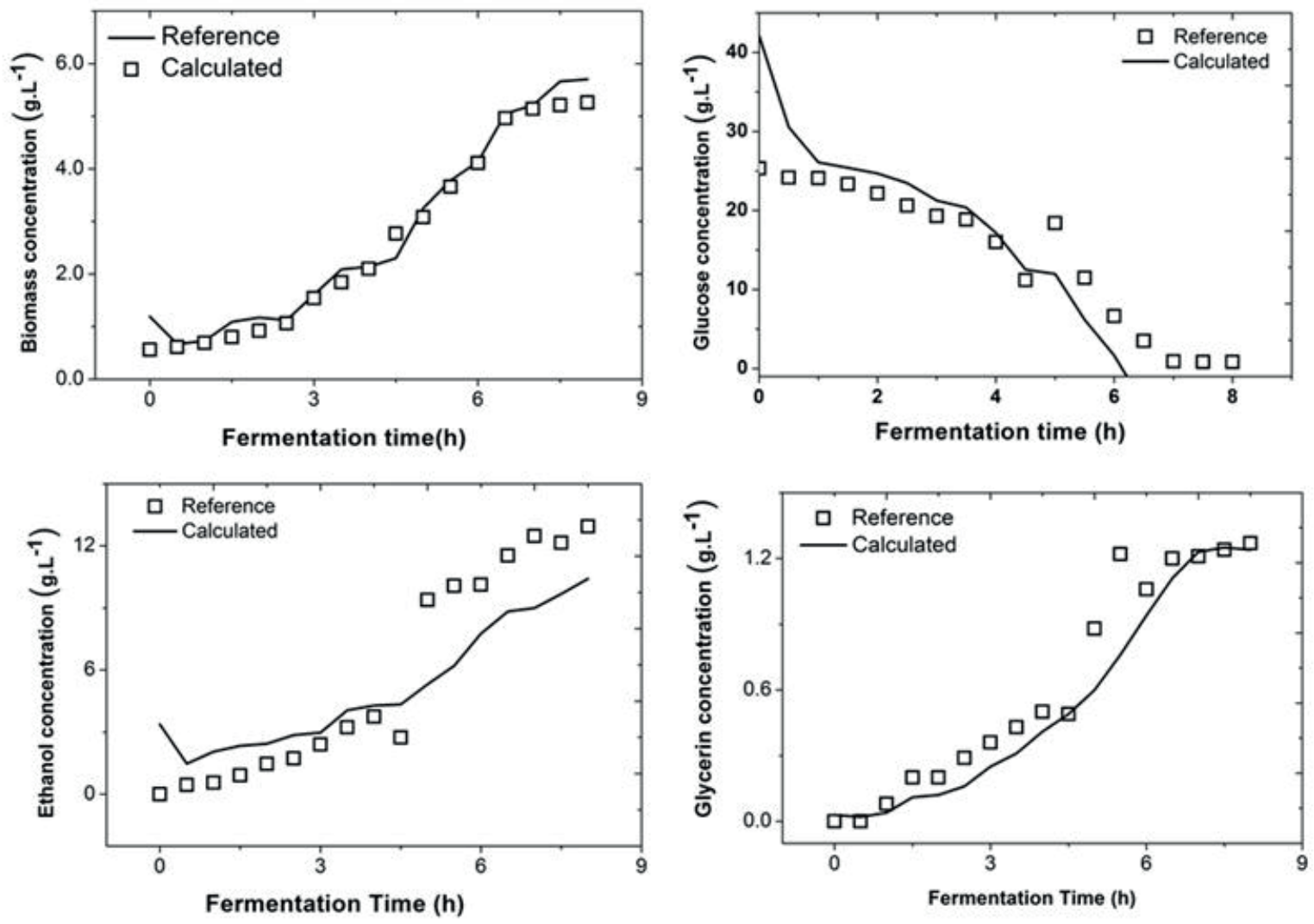

Figure 6. Kinetic curves (Batch 5) obtained by the best NIRS model. The hollow symbols denote reference concentration values and the solid lines represent predicted concentration values 


\section{CONCLUSION}

In alcoholic fermentation processes, the analyses required to monitor glucose, ethanol, glycerine and biomass concentrations are time-consuming and are performed off-line in laboratories. In addition to requiring laborious tasks, these methods are expensive and use reagents that can be detrimental to the environment. The objective of this study was to evaluate the real-time monitoring of important parameters in alcoholic fermentations using a near-infrared spectroscopy (NIRS) probe. Multivariate data analyses allowed spectra collected in real-time to be correlated with analyte concentrations obtained from reference methods. PCA allowed data to be selected for the calibration and external validation sets, and PLS regression ensured appropriate model fitting. In general, the results demonstrated that an in situ NIRS probe is suitable for monitoring important parameters of alcoholic fermentations. Analysis time was reduced drastically, as an NIR spectrum can be acquired in less than $1 \mathrm{~min}$. This fact supports the idea of real-time monitoring. It is important to highlight that a similar monitoring system may be scaled up for industrial bioreactors, and control strategies may be implemented to improve process conditions.

\section{ACKNOWLEDGMENTS}

The authors thank CAPES and CNPq for financially supporting this research.

\section{REFERENCES}

Alves-Rausch, J., Bienert, R., Grimm, C., Bergmaier, D., Real time in-line monitoring of large scale Bacillus fermentations with near-infrared spectroscopy. J. Biotechnol., 189, 120-128 (2014).

Arnold, S. A., Crowley, J., Woods, N., Harvey, L. M., McNeil, B., In-situ Near Infrared Spectroscopy to Monitor Key Analytes in Mammalian Cell Cultivation. Biotechnol. Bioeng., 84, 13-19 (2003).

Blanco, M., Peinado, A. C., Mass, J., Analytical monitoring of alcoholic fermentation using NIR spectroscopy, Biotechnol. Bioeng., 88, 536-542, (2004).

Blanco, M., Peinado, A. C., Mas, J., Elucidating the composition profiles of alcoholic fermentations by use of ALS methodology, Anal. Chim. Acta, 544, 199-205, (2005).

Blanco, M., Peinado, A. C., Mas, J., Monitoring alcoholic fermentation by joint use of soft and hard modelling methods, Anal. Chim. Acta, 556, 364-373, (2006).

Burratti, S., Ballabio, D., Giovanelli, G., Dominguez, Z., Moles, A., Benedetti, S., Sinelli, N., Monitoring of alcoholic fermentation using near infrared and mid infrared spectroscopies combined with electronic nose and electronic tongue, Anal. Chim. Acta, 697, 67-74, (2011).

Coffey, C.; Cooley, B. E. Jr.; Walker, D. S., Real time quantitation of a chemical reaction by fiber optic nearinfrared spectroscopy. Anal. Chim. Acta., 395(3), 335341 (1999).

Cordella, C. B. Y. (2012). PCA: The Basic Building Block of Chemometrics, Analytical Chemistry, Dr. Ira S. Krull (Ed.), InTech.

Cozzolino, D.; Parker, M.; Dambergs, R. G.; Herderich, M.; Gishen, M., Chemometrics and Visible-Near Infrared Spectroscopic Monitoring of Red Wine Fermentation in a Pilot Scale. Biotechnol. Bioeng., 95(6), 1101-1107 (2006).

Crowley, J., Arnold, S. A., Wood, N., Harvey, L. M., McNeil, B., Monitoring a high cell density recombinant Pichia pastoris fed-batch bioprocess using transmission and reflectance near infrared spectroscopy. Enzyme Microb. Tech., 36, 621-628 (2005).

Egidio, V. D; Sinelli, N.; Giovanelli, G.; Moles, A.; Casiraghi, E., NIR and MIR spectroscopy as rapid methods to monitor red wine fermentation. Eur. Food Res. Technol. 230(6), 947-955 (2010).

Fayolle, Ph.; Picque, D.; Corrieu, G., On-line monitoring of fermentation processes by a new remote dispersive middle-infrared spectrometer. Food Control., 11, 291296 (2000).

Fernandéz-Novales, J.; López, M. I.; Sánchez, M. T,; García, J. A.; Morales, J., A feasibility study on the use of a miniature fiber optic NIR spectrometer for the prediction of volumic mass and reducing sugars in white wine fermentations. Journal of Food Engineering, 89(3), 325-329 (2008).

Ferreira, M. M. C., Antunes, A. M., Melgo, M. S., Volpe, P. L. O., Quimiometria I: calibração multivariada, um tutorial. Quim. Nova, 22, 724-731, (1999).

Filmozer, P., Liebmann, B., Varmuza, K., Repeated double cross validation. J. Chemom., 29, 160-171 (2009).

Finn, B., Harvey, L. M., Mcneil, B., Near-infrared spectroscopic monitoring of biomass, glucose, ethanol and protein content in a high cell density baker's yeast fed-batch bioprocess, Yeast, 26, 507-517, (2006).

Geladi, P., Kowalski, B. R., Partial least-squares regression: a tutorial. Anal. Chim. Acta, 185, 1-17 (1986).

González-Sáiz, J. M.; Esteban-Díez, I.; Sánchez-Gallardo, C.; Pizarro, C., Monitoring of substrate and product concentrations in acetic fermentation processes for onion vinegar production by NIR spectroscopy: value addition to worthless onions. Anal. Bioanal. Chem., 391(8), 2937-2947 (2008).

Gonzáles-Sáiz, J. M., Pizarro, C., Estebán-Díez, I., Ramírez, O., Gonzáles-Navarro, C. J., Sáiz-Abajo, 
M. J., Itoiz, R., Monitoring of Alcoholic Fermentation of Onion Juice by NIR Spectroscopy: Valorization of Worthless Onions. J. Agric. Food Chem, 55, 2930-2936 (2007).

Hall, J. W., McNeil, B., Rollins, M. J., Draper, I., Thompson, B. G., Macaloney, G., Near-Infrared Spectroscopic Determination of Acetate, Ammonium, Biomass, and Glycerol in an Industrial Escherichia coli Fermentation. Appl. Spectroscop., 50, 102-108 (1996).

Hakemeyer, C.; Strauss, H.; Werz, S.; Folque, F.; Menezes, J. C., Near-infrared and two-dimensional fluorescence spectroscopy monitoring of monoclonal antibody fermentation media quality: Aged media decreases cell growth. Biotechnol. J., 8, 835-846 (2013).

Hongqiang, L. \& Hongzhang, C. Near-infrared spectroscopy with a fiber-optic probe for state variables determination in solid-state fermentation. Process Biochem., 43(5), 511-516 (2008).

Jiang, H., Liu, G., Xiao, X., Mei, C., Ding, Y., Yu, S., Monitoring of solid-state fermentation of wheat straw in a pilot scale using FT-NIR spectroscopy and support vector data description. Microchem J., 102, 68-74 (2012).

Liang, J., Zhang, D., Guo, X., Xu, Q., Xie, X., Zhang, C., Bai, G., Xiao, X., Chen, N., At-line nearinfrared spectroscopy for monitoring concentrations in temperature-triggered glutamate fermentation. Bioprocess Biosyst. Eng., 36, 1879-1887 (2013).

Meng-Lin, Z., Guo-Ping, S., Yang, M., Wei-Hua, L., HanQing, Y., Hideki, H., Yu-You, L., Rapid and accurate determination of VFAs and ethanol in the effluent of an anaerobic H2-producing bioreactor using near-infrared spectroscopy. Water Res., 43, 1823-1830 (2009).

Naes, T., Isaksson, T., Fearn, T., Davies, T., Multicollinearity and the need for data compression. In: Naes, T. editor. A User friendly guide to multivariate calibration and classification. Chichester, UK: NIR Publications, Chap. 4, 24-25 (2002).

Nishii, T. Genkawa, T., Watari, M., Ozaki, Y., Selection of the NIR Region for a Regression Model of the Ethanol Concentration in Fermentation Process by an Online NIR and Mid-IR Dual-Region Spectrometer and 2D Heterospectral Correlation Spectroscopy. Anal Sci., 28, 1165-1170 (2012).

Petersen, A. E. C., Petersen, N., Lantz, A. E., Larsen, A., Gernaey, K. V. Application of near-infrared spectroscopy for monitoring and control of cell culture and fermentation. Biotechnol. Progr., 25(6), 1561-1581 (2009).

Randall, T. D., An introduction to partial least squares regression. SAS institute, 1-8 (1999).

Rocha, M. V. P., Produção de bioetanol a partir de pedúnculo de caju (Anacardium occidentale L.) por fermentação submersa, D.Sc. Thesis, Federal University of Rio Grande do Norte (2010).

Roychoudhury, P., Harvey, L. M., McNeil, B., At-line monitoring of ammonium, glucose, methyl oleate and biomass in a complex antibiotic fermentation process using attenuated total reflectance-mid-infrared (ATRMIR) spectroscopy. Anal. Chim. Acta, 561, 218-224 (2006).

Svante W., Chemometrics; what do we mean with it, and what do we want from it?. Chemometr Intell Lab., 30, 109-115 (1995).

Schügerl, K., Progress in monitoring, modeling and control of bioprocesses during the last 20 years. J. Biotechnol., 85, 149-173 (2001).

Tamburini, E., Vaccari, G., Tosi, S., Trilli, A., Near-Infrared Spectroscopy: A Tool for Monitoring Submerged Fermentation Processes Using an Immersion OpticalFiber Probe. Appl. Spectrosc., 57(2), 132-138 (2003).

Tamburini, E., Vaccari, G., Tosi, S., Trilli, A., Near-Infrared Spectroscopy: A Tool for Monitoring Submerged Fermentation Processes Using an Immersion OpticalFiber Probe. Appl. Spectrosc., 57(2), 132-138 (2003).

Xie, L., Ye, X., Liu, D., Ying, Y. Quantification of glucose, fructose and sucrose in bayberry juice by NIR and PLS. Food Chemistry, 114(3), 1135-1140 (2009). 